\title{
Tunnel switch diode based on AISb/GaSb heterojunctions
}

\author{
X.-C. Cheng, X. Cartoixà, M. A. Barton, C. J. Hill, and T. C. McGill ${ }^{\mathrm{a}}$ \\ Thomas J. Watson, Sr. Laboratory of Applied Physics, California Institute of Technology, Pasadena, \\ California 91125
}

(Received 13 July 2000; accepted for publication 17 August 2000)

\begin{abstract}
We report on tunnel switch diodes based on AlSb barriers and $\mathrm{GaSb} p-n$ junctions grown by molecular beam epitaxy. These were the devices with thyristor like switching in the GaSb/AlSb system. The characteristic " $S$ " shaped current-voltage curve was found to occur for structures with AlSb barriers less than $300 \AA$ thick. The switching voltage and current density exhibited less sensitivity to barrier and epilayer thickness than was predicted by the punch-through model. The results were correlated with drift diffusion simulations which have been modified to account for the presence of a tunneling contact. C 2000 American Institute of Physics. [S0021-8979(00)06922-X]
\end{abstract}

The tunnel switch diode (TSD), also known as the metal insulator semiconductor switch (MISS) ${ }^{1}$ or controlled inversion device (CID), ${ }^{2}$ is characterized by its " $S$ " shaped current-voltage $(I-V)$ behavior. The device has unique applications in circuit design due to its large nonlinearity, inherent speed, and integration capability which arises from its vertical structure. For example, a TSD-based, single element static random access memory (SRAM) cell ${ }^{3}$ has been implemented in the $\mathrm{Si}$ system by using $\mathrm{SiO}_{2}$ as the tunnel barrier on top of a $\mathrm{Si} p-n$ junction. ${ }^{4}$ Compared to rival transistor designs, the TSD-SRAM is both more compact due to its structural simplicity and faster because of the tunneling nature of the switching process.

In this article, we demonstrate the successful implementation of the TSD in the GaSb/AlSb system. This device can be seen as the dual complement of the InAs/AlSb/GaSb resonant interband tunneling (RIT) diode ${ }^{5}$ and will add more functionality to antimonide circuits in high speed applications. ${ }^{6}$ The structural dependence of the TSD $I-V$ characteristics is examined, and the results compared to drift diffusion simulation to confirm the effect of tunneling barrier on TSD switching.

The band diagram of the antimonide TSD is shown in Fig. 1. The basic device structure consists of an unintentionally doped AlSb barrier layer in series with a GaSb $p-n$ junction. For the $p-n$ junction polarity shown, the device is forward biased when a positive voltage is applied to the AlSb barrier. Switching from high to low impedance state occurs when the depletion region in the $p$-GaSb layer punches through to the buried $p-n$ junction, or when the electric field in the depletion region becomes high enough to trigger avalanche multiplication of minority carriers in the layer. ${ }^{7}$ Due to the low doping density of the $p$-GaSb epilayer, the devices in this study are expected to operate in the punch through mode. The switching voltage is given by

$$
V_{\text {switch }}=q N_{c}\left(w-w_{0}\right)^{2} / 2 \epsilon_{\mathrm{GaSb}},
$$

where $q$ is the electronic charge, $w_{0}$ is the zero bias depletion width of the $p-n$ junction, and $N_{c}, w$, and $\epsilon_{\mathrm{GaSb}}$ are the

a)Electronic mail: tcm@ssdp.caltech.edu doping density, width, and dielectric constant of the $p$-GaSb epilayer, respectively. ${ }^{1}$ The polarity of the buried $p-n$ junction is such that the AlSb layer partially blocks the electron flow in the high impedance state. This is the desired mode of operation because AlSb is a more effective barrier for electrons than holes, i.e., the conduction band offset between $\mathrm{AlSb}$ and $\mathrm{GaSb}$ is $1.15 \mathrm{eV}$ at the $\Gamma$ point and $0.55 \mathrm{eV}$ at the $X$ point, which is larger than the valence band offset of 0.4 $\mathrm{eV}$ between these materials. The AlSb layer is typically much thicker than the oxide in Si based devices to compensate for its relative lack of barrier height.

As listed in Table I, six different wafers were grown for this study. The AlSb barrier thickness was varied in these samples to delineate its effect on switching, whereas the $p$-type GaSb layer thickness was varied among $100 \AA \mathrm{AlSb}$ barrier samples to study its effect on the switching voltage.

All samples were grown by molecular beam epitaxy on Te doped $\left(n=1 \times 10^{18} \mathrm{~cm}^{-3}\right)$ GaSb wafers. The $p-n$ junction was formed at the substrate surface by depositing an unintentionally doped $p$-type $\mathrm{GaSb}$ buffer layer $(p \approx 5$ $\left.\times 10^{16} \mathrm{~cm}^{-3}\right)$. In order to improve the quality of the $p-n$ junction, the substrate was etched and thoroughly heated for oxide desorption prior to buffer layer growth. At the end of the growth, the structure was capped by a $50 \AA \mathrm{GaSb}$ layer to prevent oxidation of the AlSb barrier. The crystal quality was monitored with high resolution $\mathrm{x}$-ray scans, which yielded a full width at half maximum (FWHM) of 18 arcsec for the GaSb epitaxial layer. The wafers were metallized $e x$ situ with $\mathrm{Au}$ in a sputter deposition tool. Indium left over from the growth served as the back contact. Device mesas were defined by standard photolithography and ranged in size from 38 to $200 \mu \mathrm{m}$. Chlorine assisted dry etching was used as the final etch down and yielded highly reproducible surfaces with few etch defects. The $I-V$ characteristics of these devices were measured by using a HP4156 semiconductor parameter analyzer.

The typical $I-V$ characteristic of the antimonide TSD with thyristor-like switching is shown in Fig. 2. As listed in Table I, switching behavior was observed for all devices with 100 and $200 \AA$ AlSb barrier thicknesses. When the AlSb barrier was increased to beyond $300 \AA$, no switching 


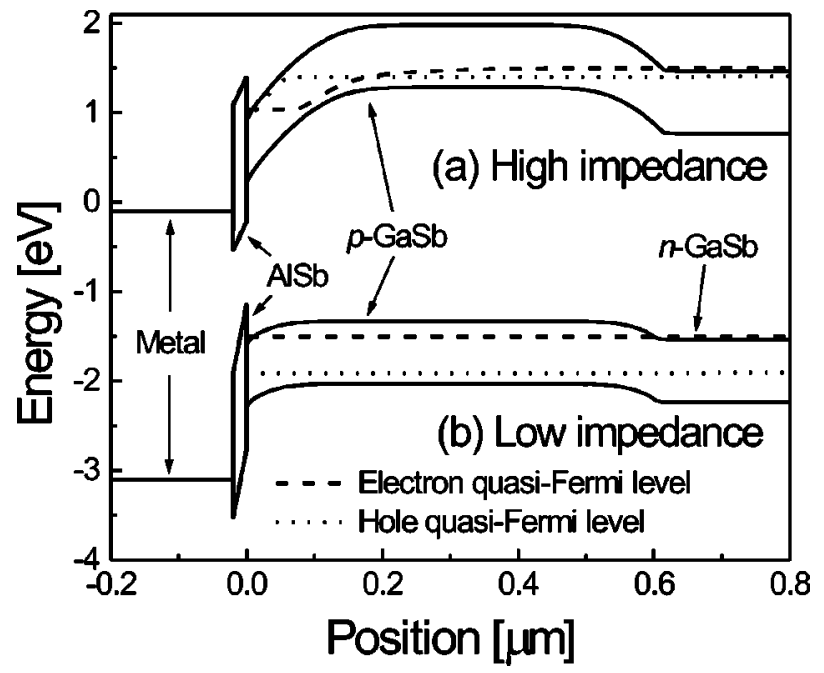

FIG. 1. Band diagrams of an antimonide TSD at a forward bias of 1.6 V. (a) High impedance state with deep depletion in the $p$-GaSb epilayer. (b) Low impedance state with the $p-n$ junction turned on and most of the bias dropped across the AlSb barrier. The energy scale of the high and low impedance states are shifted +1.5 and $-1.5 \mathrm{~V}$, respectively, for clarity.

was observed. The device instead remained in the high impedance state under forward bias up to a breakdown voltage of 5-7 V. Typical switching current densities ranged from 10 to $300 \mathrm{~A} / \mathrm{cm}^{2}$, and had a much weaker dependence on the AlSb barrier thickness than expected from theory. ${ }^{7}$ Similar insensitivity of switching current to barrier thickness has also been observed in $\mathrm{Si} / \mathrm{SiO}_{2}$ devices. ${ }^{8}$ The switching voltage ranged from 2.3 to $3.5 \mathrm{~V}$ and was relatively independent of $p$-type $\mathrm{GaSb}$ epilayer thickness. This result did not agree with the punch through model, which predicts a lower switching voltage for thinner GaSb epilayers.

Similar to Si TSDs, ${ }^{8}$ the low impedance branch of the $I-V$ curve shifted slightly upward when the device was current stressed. The breakdown current density for the TSD device was on the order of $10^{3} \mathrm{~A} / \mathrm{cm}^{2}$. Post breakdown characteristic was $p-n$ diode like, indicating that the breakdown occurred in the AlSb barrier. For a number of devices, the switching behavior was also modified following significant current stressing. As shown in Table I, the after stress switching voltage and current agglomerated around $1.2 \mathrm{~V}$ and $0.2-1 \mathrm{~mA}$. Stress induced modifications have been observed in TSDs in other material systems, ${ }^{9}$ but such agglom-

TABLE I. Antimonide TSD structures fabricated and the observed switching characteristics. Device size was $67 \mu \mathrm{m}$.

\begin{tabular}{|c|c|c|c|}
\hline \multicolumn{2}{|c|}{ Device configuration } & \multicolumn{2}{|c|}{ Switching voltage and current } \\
\hline $\begin{array}{l}\text { AlSb } \\
\text { barrier }\end{array}$ & $\begin{array}{l}p \text {-GaSb } \\
\text { epilayer }\end{array}$ & Initial values & After stressing \\
\hline $100 \AA$ & $0.2 \mu \mathrm{m}$ & $\begin{array}{c}2.3 \mathrm{~V} / 2.0 \mathrm{~mA} \\
\text { (unstable) }\end{array}$ & $1.2 \mathrm{~V} / 1.0 \mathrm{~mA}$ \\
\hline $100 \AA$ & $0.4 \mu \mathrm{m}$ & $3.5 \mathrm{~V} / 3.0 \mathrm{~mA}$ & $1.2 \mathrm{~V} / 1.0 \mathrm{~mA}$ \\
\hline $100 \AA$ & $0.6 \mu \mathrm{m}$ & $3.0 \mathrm{~V} / 2.1 \mathrm{~mA}$ & $1.3 \mathrm{~V} / 0.2 \mathrm{~mA}$ \\
\hline $200 \AA$ & $0.6 \mu \mathrm{m}$ & $3.1 \mathrm{~V} / 1.8 \mathrm{~mA}$ & $1.2 \mathrm{~V} / 0.3 \mathrm{~mA}$ \\
\hline $300 \AA$ & $0.6 \mu \mathrm{m}$ & \multicolumn{2}{|c|}{ no switching observed } \\
\hline $500 \AA$ & $0.6 \mu \mathrm{m}$ & \multicolumn{2}{|c|}{ no switching observed } \\
\hline
\end{tabular}

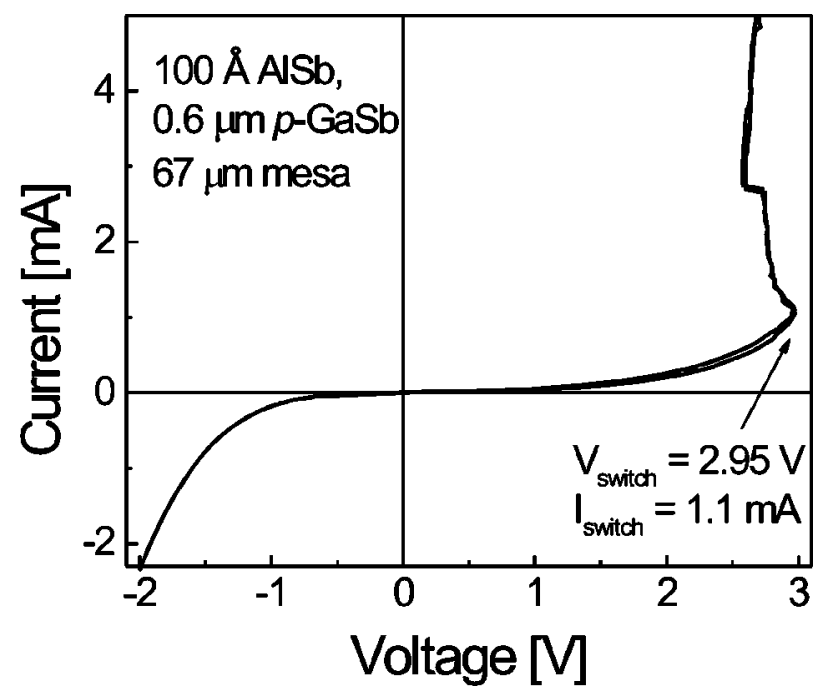

FIG. 2. Current-voltage characteristics of an antimonide TSD with a $100 \AA$ AlSb barrier and a $0.6 \mu \mathrm{m} p$-GaSb epilayer. The reverse current was large and varied exponentially with voltage, indicating that tunneling through the buried $p-n$ junction was significant. The less than ideal quality of the $p-n$ junction was expected since it was formed at the substrate surface.

eration of post stress switching voltages and currents was unique to the antimonide device. We speculate that this was due to activation of a deep level in the AlSb layer or possible modification of surface charge at the AlSb to $p$-GaSb layer interface.

Since the TSD switching was strongly dependent on the AlSb barrier thickness, we have used a computer model $^{10}$ to simulate the $I-V$ characteristics of device structures with different AlSb barriers. The simulation was based on the Poisson and the carrier continuity equations ${ }^{11}$

$$
\begin{aligned}
& \nabla^{2} \phi-\frac{q}{\epsilon}(n-p-C)=0 \\
& \nabla \cdot \mathbf{J}_{n}-q R(\phi, n, p)=0, \\
& \nabla \cdot \mathbf{J}_{p}+q R(\phi, n, p)=0
\end{aligned}
$$

with

$$
\begin{aligned}
& \mathbf{J}_{n}=q D_{n} \boldsymbol{\nabla} n-q \mu_{n} n \boldsymbol{\nabla} \phi, \\
& \mathbf{J}_{p}=-q D_{p} \boldsymbol{\nabla} p-q \mu_{p} p \boldsymbol{\nabla} \phi,
\end{aligned}
$$

where $\phi$ is the electric potential, $n$ and $p$ are the electron and hole concentrations, $q$ is the electron charge, $\epsilon$ is the semiconductor dielectric constant, $C$ is the net concentration of ionized dopants $\left(N_{d}^{+}-N_{a}^{-}\right), \mathbf{J}_{n}$ and $\mathbf{J}_{p}$ are the electron and hole current densities, $R$ is the net recombination rate, $D_{n}$ and $D_{p}$ are the electron and hole diffusion constants, and $\mu_{n}$ and $\mu_{p}$ are the electron and hole nobilities. The boundary conditions at the surface of the barrier are determined by the generation-recombination component and the tunneling current according to

$$
\begin{aligned}
& \mathbf{e}_{n} \cdot \mathbf{J}_{n}=-q R_{s}+J_{n \text { calc }}, \\
& \mathbf{e}_{n} \cdot \mathbf{J}_{p}=q R_{s}+J_{p \text { calc }},
\end{aligned}
$$




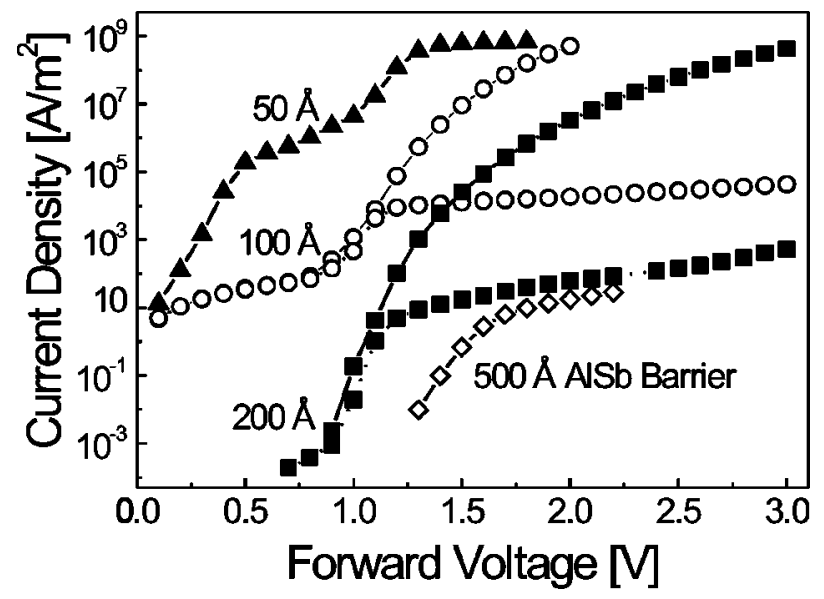

FIG. 3. Simulated characteristics for TSD devices with $0.6 \mu \mathrm{m} p$-GaSb epilayer and different AlSb thicknesses. Bistable states were obtained for 100 and $200 \AA$ barrier thicknesses. Only the low impedance state was obtained for the barrier thickness of $50 \AA$ whereas the $500 \AA$ barrier yielded only the high impedance state.

where $\mathbf{e}_{n}$ is a unit vector perpendicular to the $\mathrm{AlSb} / \mathrm{GaSb}$ interface, $R_{s}$ is the generation-recombination rate, and $J_{n \text { calc }}$ and $J_{p \text { calc }}$ are the calculated electron and hole tunneling currents, respectively.

To reduce the computational load, we have employed the Scharfetter-Gummel scheme ${ }^{11}$ to discretize the equations and the Newton-Raphson method to solve the resulting set of nonlinear algebraic equations. The multivalued nature of the TSD $I-V$ curve contributed much to the complexity of the simulation. In the negative differential resistance region, the device presented two stable current values for a fixed voltage, hence the convergence of the simulation to a particular solution was strongly dependent on the initial condition.

Figure 3 shows the simulated $I-V$ characteristics of TSD devices with AlSb barriers ranging from 50 to $500 \AA$. It can be seen that only the low impedance state existed for the $50 \AA \mathrm{AlSb}$ barrier device whereas the $500 \AA$ barrier device yielded only the high impedance state. Dual impedance state behavior was obtained for AlSb barrier thicknesses of 100 and $200 \AA$, which agreed with experimental findings.

In summary, we have fabricated the antimonide tunnel switch diode and observed successful operation of the device. Switching was obtained for AlSb barrier thicknesses between 100 and $300 \AA$, as was predicted by drift diffusion simulations. The switching voltage and current density had a weak dependence on the barrier and epilayer thickness and did not agree with the punch-through model. Modification of the switching behavior following current stressing was also observed and was attributed to instability associated with the AlSb layer. These deviations from the expected behavior indicate the importance of developing a better AlSb layer and $\mathrm{AlSb} / \mathrm{GaSb}$ interface.

The authors thank E. S. Daniel, D. Z.-Y. Ting, W. Frensley, and K. Smith for their fruitful discussions on TSD simulations. This work is supported in part by the Office of Naval Research under Grant No. N00014-98-1-0567.

${ }^{1}$ J. S. Simmons and A. El-Badry, Solid-State Electron. 20, 955 (1977).

${ }^{2}$ H. Kroger and H. A. R. Wegener, Appl. Phys. Lett. 27, 303 (1975).

${ }^{3}$ H. J. Levy and T. C. McGill, US Patent 5,535,156 (1996).

${ }^{4}$ T. Yamamoto and M. Morimoto, Appl. Phys. Lett. 20, 269 (1972).

${ }^{5}$ J. R. Söderström, D. H. Chow, and T. C. McGill, Appl. Phys. Lett. 55, 1094 (1989)

${ }^{6}$ D. H. Chow, H. L. Dunlap, W. Williamson, III, S. Enquist, B. K. Gilbert, S. Subramaniam, P.-M. Lei, and G. H. Bernstein, IEEE Electron Device Lett. 17, 69 (1996).

${ }^{7}$ S. E.-D. Habib and J. G. Simmons, Solid-State Electron. 22, 181 (1979).

${ }^{8}$ P. O. Pettersson, A. Zur, E. S. Daniel, H. J. Levy, O. J. Marsh, and T. C. McGill, IEEE Trans. Electron Devices 45, 286 (1998).

${ }^{9}$ E. S. Daniel, PhD thesis, Caltech, November 1997.

${ }^{10}$ E. S. Daniel, X. Cartoixà, W. R. Frensley, D. Z.-T. Ting, and T. C. McGill, IEEE Trans. Electron Devices 47, 1052 (2000).

${ }^{11}$ S. Selberherr, Analysis and Simulation of Semiconductor Devices (Springer, Wien, Austria, 1984) 